\title{
Trans-splicing and polyadenylation of let-7 microRNA primary transcripts
}

\author{
JOHN BRACHT, ${ }^{1}$ SHAUN HUNTER, ${ }^{1}$ RACHEL EACHUS, PHILLIP WEEKS, and AMY E. PASQUINELLI \\ Department of Biology, University of California, San Diego, La Jolla, California 92093, USA
}

\begin{abstract}
Members of the microRNA (miRNA) class of 22-nucleotide RNAs regulate the expression of target genes that contain sequences of antisense complementarity. Maturation of miRNAs involves cleavage of longer primary transcripts, but little is yet understood about how miRNA genes are transcribed and enter the processing pathway. We find that relatively long, polyadenylated transcripts encoded by the Caenorhabditis elegans let-7 gene undergo trans-splicing to the spliced leader 1 (SL1) RNA. Deletions, including removal of the trans-splice site, upstream of mature let-7 sequence result in stable accumulation of primary transcripts and compromised production of mature let-7 RNA in vivo. Our data show that multiple steps of let-7 miRNA biogenesis can be uncoupled, allowing for complex regulation in the production of a functional miRNA. Finally, the observation that let-7 primary transcripts undergo splicing highlights the importance of identifying the sequence of endogenous pri-miRNA substrates recognized by the cellular processing machinery.
\end{abstract}

Keywords: microRNAs, let-7, trans-splicing, polyadenylation; miRNAs

\section{INTRODUCTION}

The newly established class of regulatory RNAs, called microRNAs (miRNAs), is comprised of $\sim 22$-nucleotide RNAs that control expression of target genes via antisense basepairing (Lai 2003; Bartel 2004). All multicellular organisms are believed to express miRNAs and the Caenorhabditis elegans, Drosophila, and human genomes each have been predicted to encode more than 100 different miRNAs (Grad et al. 2003; Lai et al. 2003; Lim et al. 2003a,b). Already members of this new class of tiny regulatory RNAs have been implicated in diverse biological roles, including temporal patterning, cell death regulation, and control of fat metabolism (Ambros 2003; Bartel 2004).

The founding members of the microRNA gene family, lin-4 and let-7, were identified as essential regulators of temporal development in the nematode C. elegans (Pasquinelli and Ruvkun 2002). The developmental function of lin-4 and let-7 RNAs is related to the timing of their expression: Mature lin-4 RNA first appears midway through

\footnotetext{
${ }^{1}$ These authors contributed equally.

Reprint requests to: Amy E. Pasquinelli, Department of Biology, University of California, San Diego, La Jolla, CA 92093, USA; e-mail: apasquin@ucsd.edu. fax: (858) 822-3021.

Article published online ahead of print. Article and publication date are at http://www.rnajournal.org/cgi/doi/10.1261/rna.7122604.
}

the first larval stage to regulate early larval cell divisions (Lee et al. 1993; Feinbaum and Ambros 1999), and mature let-7 RNA is undetectable until the last larval stages when it is needed to direct larval to adult cellular transitions (Reinhart et al. 2000). The let-7 RNA sequence, its general temporal expression pattern, proteins involved in its maturation, and genes controlled by this small RNA are conserved from nematodes to humans (Pasquinelli et al. 2000; Grishok et al. 2001; Hutvagner et al. 2001; Ketting et al. 2001).

The Dicer RNase processes let-7, and likely all other miRNA precursors (pre-miRNAs), to the mature $22-n t$ forms (Grishok et al. 2001; Hutvagner et al. 2001; Ketting et al. 2001; Lee et al. 2003; Lund et al. 2004). Members of the extensive and highly conserved PAZ (Piwi, Argonaute, Zwille) /Piwi Domain (PPD) protein family (Cerutti et al. 2000; Schwarz and Zamore 2002) also function in the maturation of miRNAs (Grishok et al. 2001; Hutvagner and Zamore 2002; Mourelatos et al. 2002). The nuclear export receptor Exportin-5 is responsible for delivering the $\sim 65-n t$ stem-loop structured pre-miRNAs to the cytoplasm for this maturation step (Yi et al. 2003; Bohnsack et al. 2004; Lund et al. 2004). Longer primary transcripts, called pri-miRNAs, serve as the initial substrates for processing by the nuclear Drosha RNase to generate the miRNA precursor forms (Lee et al. 2002; Basyuk et al. 2003; Lee et al. 2003; Zeng and Cullen 2003). Although sequences outside of the premiRNA hairpin have been shown to be important for RNA 
processing or stability (Lee et al. 2003; Zeng and Cullen 2003; Chen et al. 2004), little is yet known about the actual composition of pri-miRNAs.

Hundreds of miRNAs have now been isolated from diverse species, but the genomic codes that signal their production are yet to be defined. Some miRNA sequences are embedded in protein coding genes and, thus, may rely on the host gene regulatory elements for transcription (Aravin et al. 2003; Lagos-Quintana et al. 2003; Lai et al. 2003; Lim et al. 2003b). For many miRNA genes, though, there is no evidence for coexpression with messenger RNAs (mRNAs) and, thus, transcription is predicted to be directed by independent promoter elements. Regulatory motifs in miRNA genes that specify transcription by a particular polymerase have remained, for the most part, elusive. There are numerous examples of temporally or spatially restricted miRNAs, which is suggestive of Pol-II-directed transcriptional regulation. The temporal regulatory element (TRE) in the C. elegans let-7 gene can direct developmentally controlled expression of a protein-coding gene (Johnson et al. 2003), implicating Pol-II-mediated synthesis from this promoter. Nonetheless, Pol III promoters have been used to produce functional miRNAs ectopically (Zeng and Cullen 2003; Chen et al. 2004). Determination of the natural transcriptional start sites may facilitate the identification of important elements controlling expression of miRNA genes.

In this article we report the identification of the complete primary transcripts for the let-7 miRNA in C. elegans. We define two polyadenylated transcripts that likely serve as substrates for a trans-splicing reaction that appends Spliced Leader 1 (SL1) sequence to the $5^{\prime}$ end of the let-7 primiRNA. We present evidence that trans-splicing is important for generation of mature let-7 RNA and that the SL1appended form of let-7 is a substrate for processing by a $C$. elegans Drosha homolog. In contrast, factors involved in the terminal steps of let-7 maturation and stabilization, the dicer ( $d c r-1)$ and argonaute (alg-1) homologs, do not influence the transcription or initial processing events. These data identify cis-acting sequences and trans-acting factors important for specific steps of let-7 miRNA biogenesis.

\section{RESULTS}

\section{The let-7 miRNA gene encodes long, polyadenylated primary transcripts}

In C. elegans, 2460 nt of genomic DNA sequence containing let-7 is sufficient to fully rescue the let-7(mn112) putative null mutant of this gene (Reinhart et al. 2000). Prior to this study, the 65-nt precursor and 22-nt mature RNAs were the only transcripts detected from the let-7 gene. The let-7 pri-miRNAs may be rapidly processed and difficult to detect by standard Northern analyses. Thus, we employed reverse transcription polymerase chain reaction (RT-PCR) methods to identify potential let-7 pri-miRNAs. Using a let-7-specific primer in the first strand RT reaction followed by PCR with forward and reverse primers corresponding to this miRNA gene, we detected the expected size product from wild-type adult but not egg-stage RNA preparations (Fig. 1A, lanes 3,4). No product was amplified in controls where the reverse transcriptase was omitted, verifying that the PCR product is dependent on first-strand synthesis of the RNA template (Fig. 1A, lanes 6-8). Interestingly, RTPCR reactions using adult stage RNA from let-7(mn112) mutants, which harbor a 193-nt deletion upstream and including the first nucleotide of mature let-7 RNA, produced the expected size band at an apparently higher level relative to that produced from wild-type RNA (Fig. 1A, cf. lanes 4 and 5).

One hundred nucleotides upstream of the $3^{\prime}$ terminus of the 2460-nt let-7 rescue fragment is a canonical polyadenylation signal (AATAAA), raising the possibility that let-7 primary transcripts contain poly(A) tails. To test this hypothesis, total RNA preparations were subjected to RT using oligo deoxythymidine (oligo dT) as the primer for firststrand synthesis, which is expected to prime polyadenylated transcripts. The RT reactions were subjected to PCR using primers complementary to let-7 and a control mRNA transcript, translation elongation factor 2 (eft-2). Similar levels of the eft-2 product were detected in all samples, but the let-7 product in the same PCR reactions was once again undetectable in egg stage and several-fold more concentrated in adult RNA from the let-7(mn112) mutant strain compared to wild type (Fig. 1B, lanes 2-4). We conclude that long, polyadenylated let-7 transcripts may serve as substrates for the production of mature 22-nt let-7 in later stage worms. Furthermore, the lesion in the let-7(mn112) allele may inhibit processing to the 22-nt form and, thus, result in accumulation of primary transcripts.

Based on our finding that let-7 transcripts contain poly(A) tails, we predicted that these RNAs might also have $5^{\prime}$ cap structures that would facilitate identification of the $5^{\prime}$ and $3^{\prime}$ termini by standard rapid amplification of cDNA ends (RACE) methods. From wild-type RNA samples, three $5^{\prime}$ and one $3^{\prime}$ RACE products were readily detected (Fig. $1 \mathrm{C}$, lanes 2,7). Cloning and sequencing of these products revealed a common $3^{\prime}$ end approximately 70 nt downstream from the $\operatorname{poly}(\mathrm{A})$ signal that likely represents the cleavage site (Fig. 1D). The 5' RACE revealed transcripts of $\sim 1731 \mathrm{nt}$ and $\sim 890 \mathrm{nt}$ that initiate $223 \mathrm{nt}$ and $1064 \mathrm{nt}$ downstream from the temporal regulatory element (TRE; Johnson et al. 2003). We also identified a 728-nt transcript that terminates precisely $38 \mathrm{nt}$ upstream of the let-7 precursor sequence and is appended to $22 \mathrm{nt}$ from the $5^{\prime}$ end of the spliced leader 1 (SL1) RNA (Fig. 1D). The sequences and positions relative to mature let-7 RNA of the $3^{\prime}$ splice consensus sequence (TTTTCAG|G) recognized for transsplicing by SL1 and the polyadenylation site are conserved in the Caenorhabditis briggsae let-7 gene. 
A

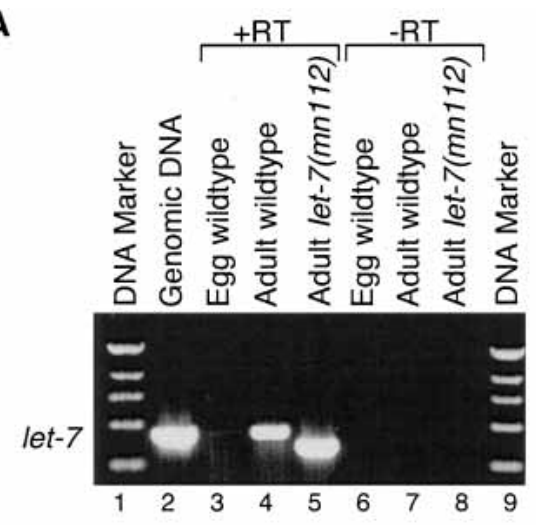

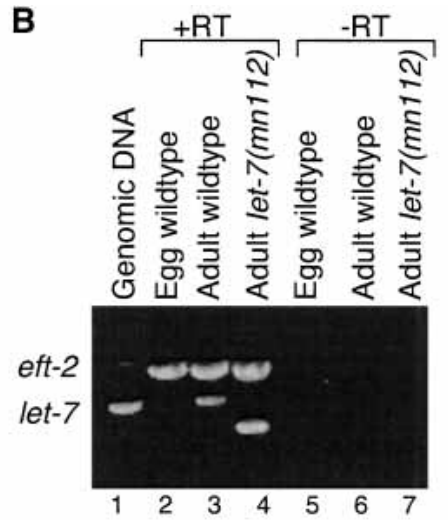

C

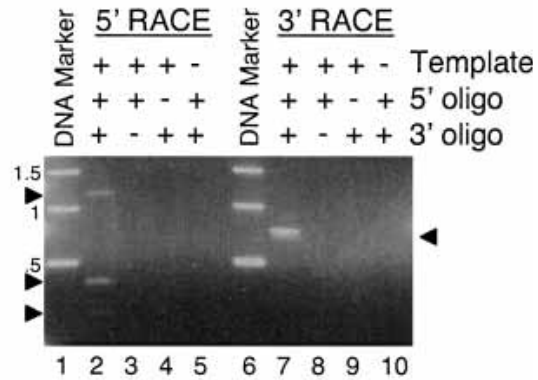

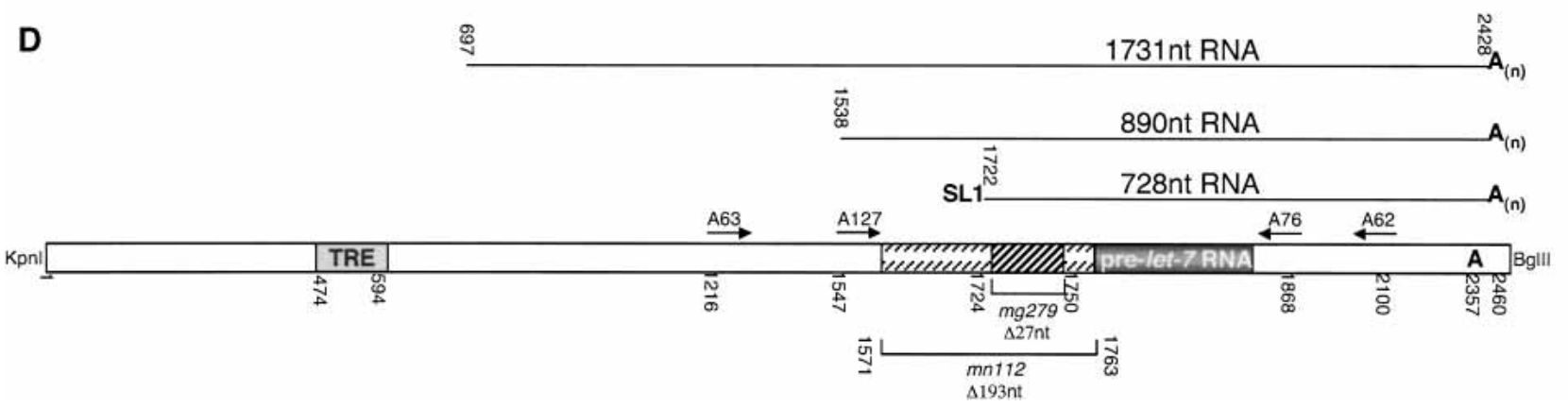

FIGURE 1. Identification of let-7 primary transcripts. (A) Total RNA samples from egg and adult stage wild-type worms and adult let-7(mn112) were subjected to reverse transcription (+RT, lanes 3-5) or mock (-RT, lanes 6-8) reactions using A62 primer (see D), followed by PCR with A63 + A62 primers. The products were separated by electrophoresis in a 1\% agarose gel and visualized by ethidium bromide staining. PCR of wild-type genomic DNA (lane 2) served as a size marker and specificity control for the PCR reactions. Lanes 1 and 9 show the migration of bands in a 1-kb DNA marker (NEB). (B) RT-PCR was performed as in A except that oligo dT primer was used for RT, and let-7-specific primers

$(\mathrm{A} 63+\mathrm{A} 76)$ and primers for eft-2 mRNA were used in the PCR. (C) Three DNA products from $5^{\prime}$ RACE and one from 3' RACE, indicated by arrowheads, were specifically amplified in reactions containing template from wild-type worms. $(D)$ The 2460-nt C. elegans genomic fragment of DNA encoding the let-7 transcripts. The deletions immediately upstream of the $\sim 65$-nt let-7 RNA precursor in let-7(mg279) and let-7(mn112) mutant strains are depicted with brackets. The positions and names of primers used in this study are indicated with arrows.

\section{Trans-splicing is important for generation of mature let-7 RNA}

Deletions upstream of mature let-7 RNA sequence were originally predicted to interfere with transcription of the RNA gene because less 22-nt let-7 RNA is observed in such mutant backgrounds (Reinhart et al. 2000). The let7(mg279) (27-nt deletion) or let-7(mn112) (193-nt deletion; see Fig. 1D) mutant strains produce several-fold less or undetectable levels of mature 22-nt let-7 RNA, respectively (Fig. 2A). In contrast, significantly increased levels of longer let-7 transcripts are observed in these deletion mutants compared to wild type, as detected by Northern blot analyses (Fig. 2B). Likewise, RT-PCR experiments using forward primers to detect only the longest transcript (Fig. 2C, first panel) or both the $\sim 1731$-nt and $\sim 890$-nt transcripts (Fig. $2 \mathrm{C}$, second panel) indicate increased levels of these RNA species in the deletion mutants relative to that observed in wild-type worms.

Recent studies have shown that sequences and structures flanking the miRNA precursor hairpin are important for maturation (Lee et al. 2003; Zeng and Cullen 2003; Chen et al. 2004). The let-7(mn112) deletion disrupts the sequence and predicted structure immediately upstream of the let-7 hairpin, supporting the idea that elements outside of the precursor sequence participate in processing recognition (Lee et al. 2003; Zeng and Cullen 2003; Chen et al. 2004). The 27-nt lesion in let-7(mg279) is located $13 \mathrm{nt}$ upstream of the let-7 hairpin and is predicted to support the same five flanking base pairs as the wild-type sequence (Fig. 3A), fitting predictions of structural requirements for primiRNA processing (Lee et al. 2003; Zeng and Cullen 2003; Chen et al. 2004). The sequence or structural alterations in let-7(mg279) upstream of the extended stem could interfere with recognition of the primary transcripts for processing to mature let-7 RNA. Additionally, the let-7(mg279) lesion appears to compromise trans-splicing. RNA from wild-type worms yields several-fold more SL1-let-7 RNA compared to that from the let-7(mg279) deletion mutant, which maintains the canonical splice sequence but alters the context of 


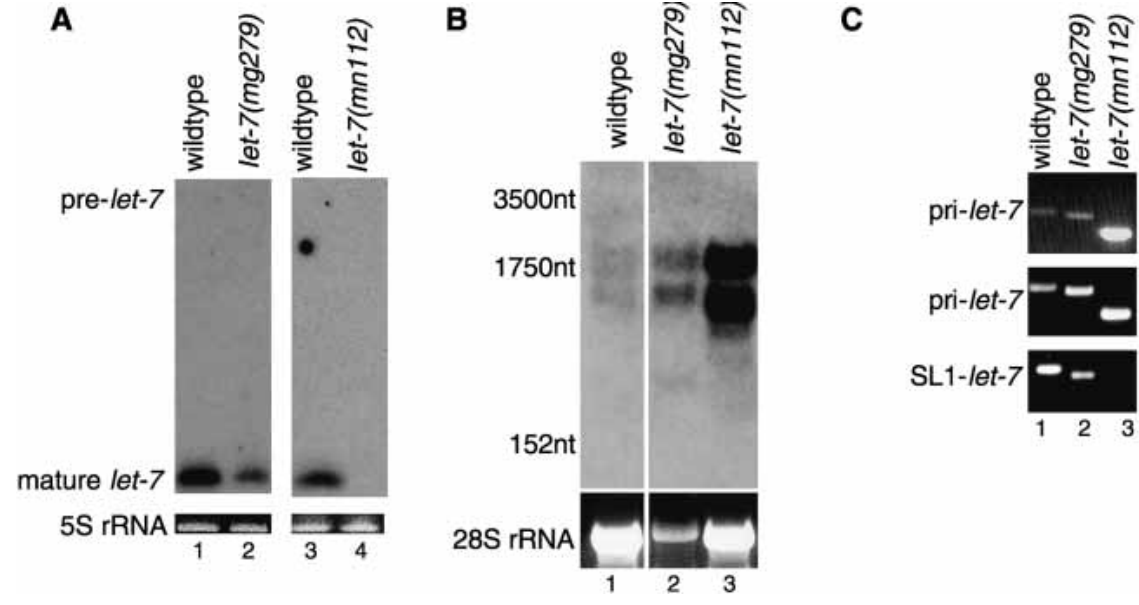

FIGURE 2. Expression of mature let-7 RNA correlates with production of SL1-spliced let-7 RNA. (A) Total RNA from adult stage wild-type, let-7(mg279) and let-7(mn112) worms was isolated, separated by $11 \%$ denaturing PAGE, and probed for precursor and mature let-7 RNA levels by Northern analyses. 5S rRNA detected by ethidium bromide staining of the gel serves as a control for the loading and quality of the RNA samples. (B) The same RNA samples as $A$ were separated under denaturing conditions in a $1.2 \%$ agarose gel and probed for longer let-7 transcripts. Positions of the 3500-nt 28S, 1750-nt 18S, and 152-nt 5.8S rRNAs are indicated. The $28 \mathrm{~S}$ rRNA detected by ethidium bromide staining is shown as a loading control. Note that the let-7(mg279) sample appears underloaded but, nonetheless, the level of long let-7 transcripts detected in this mutant still greatly exceeds that detected in wild-type worms. A lighter exposure of the abundant signal in the let-7(mn112) lane shows a faster mobility for both bands compared to the position of the bands detected in RNA from wild type, as expected to account for the 193-nt deletion in the let-7(mn112) gene. (C) The same RNA samples as in A were subjected to RT-PCR using oligo dT in the RT reaction and the let-7-specific primers A63 + A62 (first panel), A127 + A62 (second panel), or forward primer consisting of the SL1 sequence plus A62 (third panel) for PCR.

this site relative to wild type (Fig. 2C, third panel). Consistent with the absence of the $3^{\prime}$ splice site in the let-7(mn112) allele, no detectable SL1-let-7 RNA was produced from this gene (Fig. 2C, third panel). Accumulation of the $\sim 1731$-nt and $~ 890-n t$ transcripts coupled with decreased production of SL1-let-7 and 22-nt let-7 RNAs in the deletion mutants supports the idea that the longer RNAs serve as primary transcripts and that efficient trans-splicing might contribute to maturation of let-7 RNA in C. elegans.

Splicing of the SL1 RNA to let-7 primary transcripts is predicted to alter the structure adjacent to the precursor hairpin (Fig. 3, cf. boxed regions in A and B). The 3' splice site and potential for base-pairing to appended SL1 sequences are conserved in the C. elegans and C. briggsae let-7 genes. To test whether trans-splicing is important for efficient production of let-7 in vivo, we deleted $7 \mathrm{nt}$ comprising the $3^{\prime}$ splice site in the let-7 rescue fragment and asked whether this construct retained the ability to rescue the let-7(mn112) mutant strain. As previously demonstrated, the 2460-nt let-7 genomic fragment was sufficient to rescue the premature lethality of homozygous let-7(mn112) worms (Reinhart et al. 2000). In contrast, the fragment lacking only the $3^{\prime}$ splice site failed to show any rescuing activity. We confirmed that let-7 primary transcripts were synthesized from the $\Delta 3^{\prime}$ ss construct by RT-PCR of transgenic animals (data not shown). These data coupled with the observation that inefficient trans-splicing of let-7(mg279) correlates with decreased levels of mature let-7 RNA supports the possibility that the SL1-appended form of let-7 contributes to efficient maturation.

\section{Temporally regulated transcription and processing of let-7 primary transcripts}

Accumulation and trans-splicing of the predicted let-7 primary transcripts precede the appearance of mature let-7 RNA. In developmentally staged RNA samples, the 22-nt let-7 RNA first appears late in the third larval stage (L3), accumulates in L4, and persists to adulthood (Fig. 4A; Reinhart et al. 2000; Johnson et al. 2003). Similarly, the let-7 primary transcripts and SL1-spliced form were virtually undetectable by RTPCR analyses until the L3 stage of development (Fig. 4B). The relatively higher levels of these transcripts at L3 compared to later stages indicates that there may be a burst of transcriptional activity in L3 and subsequent processing that produces mature let-7 RNA that is maintained through the last larval and adult stages.

\section{The role of trans-acting factors in the biogenesis of let-7 RNA in vivo}

The ribonuclease III-type enzymes Drosha and Dicer and members of the Argonaute family function to produce mature miRNAs (Grishok et al. 2001; Hutvagner et al. 2001; Ketting et al. 2001; Hutvagner and Zamore 2002; Mourelatos et al. 2002; Lee et al. 2003). We tested whether depletion of these factors would affect the expression of the primary let-7 transcripts that enter the processing pathway. Drosha is a nuclear RNase required for efficient production of precursor miRNAs from primary transcripts in human cells (Lee et al. 2003). The C. elegans gene F26E4.10 is a predicted homolog of Drosha (Lee et al. 2003). If F26E4.10 processes miRNA primary transcripts, then let-7 primiRNAs might accumulate upon depletion of this putative RNase. In contrast, the RNase III Dicer, encoded by $d c r-1$ in C. elegans, functions downstream from Drosha processing and, thus, loss of its activity should not affect processing of let-7 primary transcripts. The rrf-3(pk1426) C. elegans strain, which is hypersensitive to RNA interference (RNAi; Simmer et al. 2002), was subjected to RNAi targeting F26E4.10 or $d c r-1$. This procedure resulted in the specific 


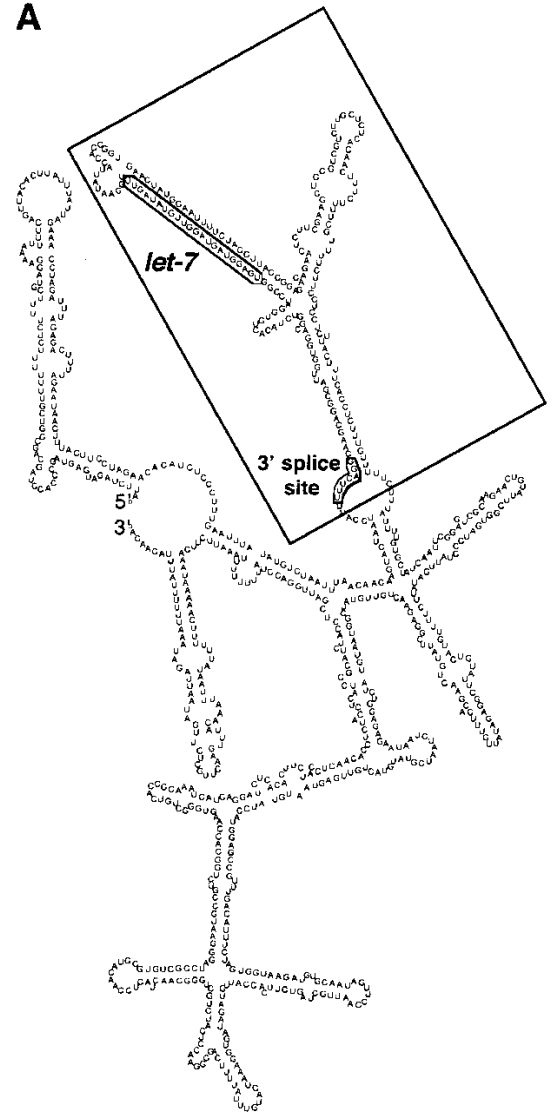

890nt pri-let-7 RNA

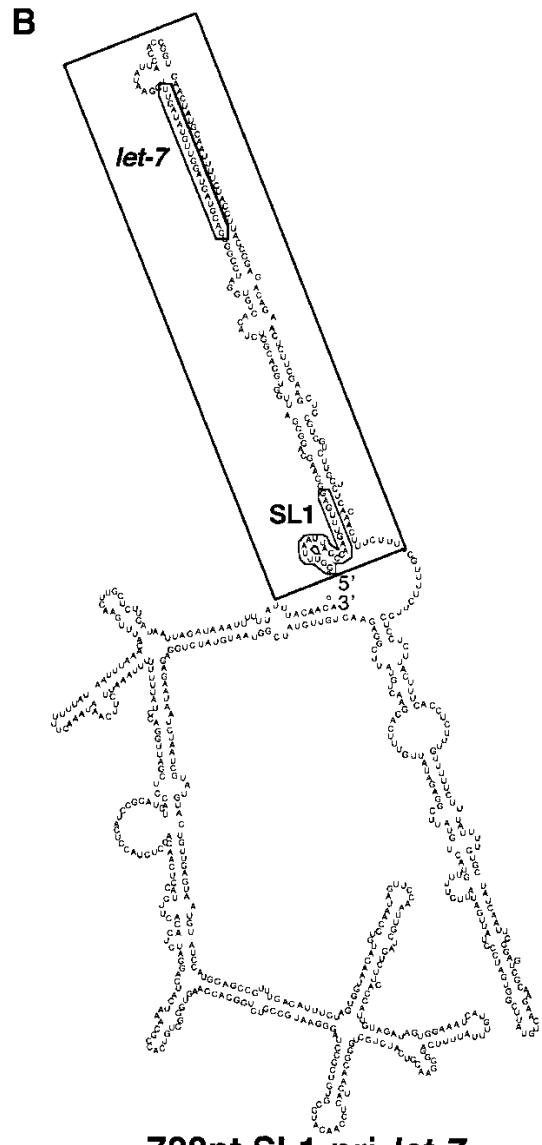

728nt SL1-pri-let-7

FIGURE 3. Predicted structures of primary and SL1-spliced let-7 transcripts. (A) Potential secondary structures of the 890-nt primary let-7 transcript and (B) the SL1-spliced RNA were generated using the mfold program (Zuker 2003). The structures shown are the lowest freeenergy predictions. The SL1 splice site, SL1 donor sequence, and mature let-7 RNA are outlined and labeled. The boxes indicate predicted structural rearrangements surrounding the let-7 stem loop region resulting from SL1 splicing.

reduction of $d c r-1$ or F26E4.10 mRNA levels (Fig. 5A). RNAi depletion of F26E4.10 or dcr-1 apparently had no effect on expression of unspliced let-7 primary transcripts (Fig. 5A, pri-let-7 panel). As expected, worms undergoing RNAi against $d c r-1$ accumulated let-7 precursor RNA (Fig. $5 \mathrm{~A}$, pre-let-7 panel). Interestingly, an increase in the SL1let-7 product was specifically observed in worms depleted of F26E4.10 (Fig. 5A, SL1-let-7 panel), indicating that the trans-spliced form of let-7 is a substrate for processing by the putative Drosha homolog.

Previously, Grishok et al. (2001) demonstrated that the argonaute-like gene alg-1 is important for accumulation of mature miRNAs. Depletion of alg-1 by RNAi results in diminished levels of 22-nt let-7 RNA without an apparent buildup of the precursor form (Grishok et al. 2001). Thus, alg-1 was proposed to be important for the stabilization of the precursors and/or mature forms of let-7 (Grishok et al. 2001). If alg-1 primarily functions at the final steps of let-7 biogenesis, then the nuclear synthesis and initial processing steps of this pathway are predicted to be unaffected by alg- 1 depletion. RNAi of alg-1 potently reduced alg-1 mRNA levels, which resulted in diminished mature let-7 RNA levels (Fig. 5B). However, relative amounts of the primary and the SL1spliced forms of let-7 RNAs were indistinguishable from worms depleted of alg- 1 compared to controls (Fig. 5B, prilet-7 and SL1-let-7 panels). We conclude that the inability to accumulate mature let-7 RNA caused by alg-1(RNAi) is independent of transcription or the initial processing of let-7 pri-miRNAs.

\section{DISCUSSION}

\section{Transcription of let-7 primary RNAs}

This study reports one of the first complete primary transcripts for an animal microRNA gene. The proposal that the $~ 1731$-nt and 890-nt RNAs serve as let-7 primary transcripts is supported by the observation that these RNAs stably accumulate in let-7 deletion mutants that produce compromised levels of mature let-7. Additionally, the timing of expression of the primary transcripts correlates well with the temporal appearance of 22-nt let-7 RNA in vivo.

The let-7 primary transcripts in C. elegans have the hallmarks of Pol-II-directed synthesis-they are apparently $5^{\prime}$-end capped, $3^{\prime}$-end polyadenylated, and substrates for splicing. However, it is yet to be experimentally determined which polymerase is responsible for transcribing let-7 or any miRNA gene. A recent report from the Slack laboratory defined a regulatory element responsible for restricting let-7 expression in the hypodermis to the last larval and adult stages (Johnson et al. 2003). A 116-bp region located $\sim 1200 \mathrm{nt}$ upstream of the mature let-7 RNA coding sequence was found to be essential for full let-7 activity. Interestingly, this region, called the temporal regulatory element (TRE), can also restrict hypodermal expression of a protein-coding gene to the same time in development as the appearance of let-7 RNA (Johnson et al. 2003). The TRE is $\sim 200 \mathrm{nt}$ upstream of the initiation site for transcription of the longest let-7 primary transcript. It remains to be determined whether the TRE regulates the expression of either or both of the endogenous pri-let-7 transcripts. In addition to the TRE, the let-7 genes in C. elegans and $C$. briggsae also share a highly homologous region $\sim 200 \mathrm{nt}$ upstream of the transcriptional start site for the $~ 890-\mathrm{nt}$ pri-let-7 RNA. Perhaps independent regulatory elements af- 


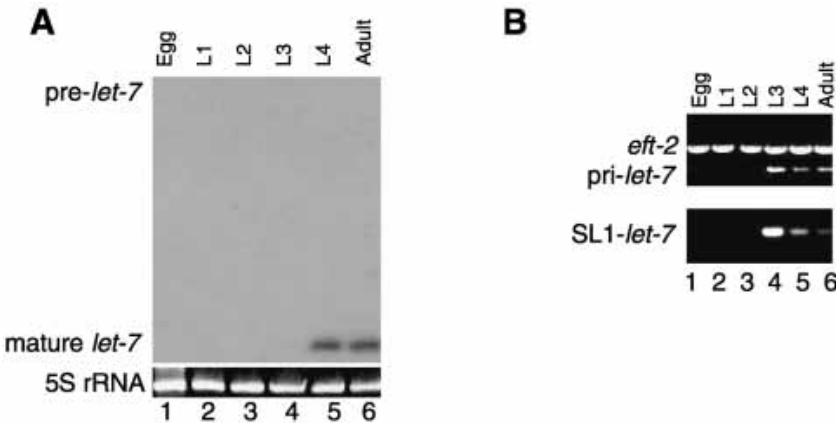

FIGURE 4. Temporally regulated expression of let-7 RNAs. (A) Total RNA from developmentally staged wild-type worms was subjected to Northern analyses to detect the precursor and mature let-7 RNAs as described in Figure 2A. (B) The same RNA samples as in $A$ were subjected to RT-PCR to detect let-7 primary transcript and control eft-2 mRNA (upper panel) or the SL1-appended let-7 RNA (lower panel).

ford tissue as well as temporally regulated transcription of let-7 RNAs.

Our demonstration that let-7 primary transcripts in $C$. elegans are polyadenylated and undergo splicing provides additional support to the indirect evidence that at least some miRNAs are transcribed by Pol II (Bartel 2004): Predicted miRNA promoter regions can produce temporally or spatially regulated expression of proteins (Johnson et al. 2003; Johnston and Hobert 2003), some miRNA sequences match ESTs (Lagos-Quintana et al. 2002; Aukerman and Sakai 2003; Smalheiser 2003), long miRNA primary transcripts might not be supported by Pol III, and some miRNAs can be processed from heterologous Pol II synthesized transcripts (Zeng et al. 2002; Zeng and Cullen 2003). If Pol II generally directs expression of miRNA genes, then how are pri-miRNA transcripts distinguished from messenger RNAs (mRNAs) in the cell? Usually, capping, splicing, and polyadenylation events facilitate export of mRNAs to the cytoplasm for translation to protein (Reed 2003). PrimiRNAs may contain sequence or structural elements that direct them to the nuclear processing pathways instead of the mRNA export route. For some miRNAs, efficient excision of the precursor hairpin may be sufficient to circumvent export of the pri-miRNA substrate.

\section{Stepwise maturation of let-7 miRNA}

The elaborate transcription and processing steps expended to eventually yield a 22-nt RNA could provide additional levels for regulation. Alteration of pri-miRNA sequence or structure beyond the precursor hairpin can affect the processing or stability of the RNA in vitro or in vivo when expressed ectopically from a transgene (Lee et al. 2003; Zeng and Cullen 2003; Chen et al. 2004). Additionally, depletion of Drosha, Dicer, Argonaute, and Exportin-5 proteins can interfere with specific steps of miRNA biogenesis (Grishok et al. 2001; Hutvagner et al. 2001; Ketting et al. 2001; Lee et al. 2003; Yi et al. 2003; Bohnsack et al. 2004; Lund et al. 2004). We show that the let-7 primary transcripts, SL1appended form, and precursor RNA can each stably accumulate in vivo when distinct processing steps are blocked by alteration of the RNA sequence or by depletion of transacting factors (Figs. 1, 2, 5), contributing to the proposal that miRNA expression can be regulated at the level of processing (Lee et al. 2002, 2003). For example, the mature form of C. elegans miR-38 is primarily observed in embryos but its precursor is detectable at all stages of development (Ambros et al. 2003).

The specific accumulation of the SL1-spliced form of let-7 upon depletion of the predicted C. elegans Drosha homolog, F26E4.10 (Fig. 5A), indicates that this form of

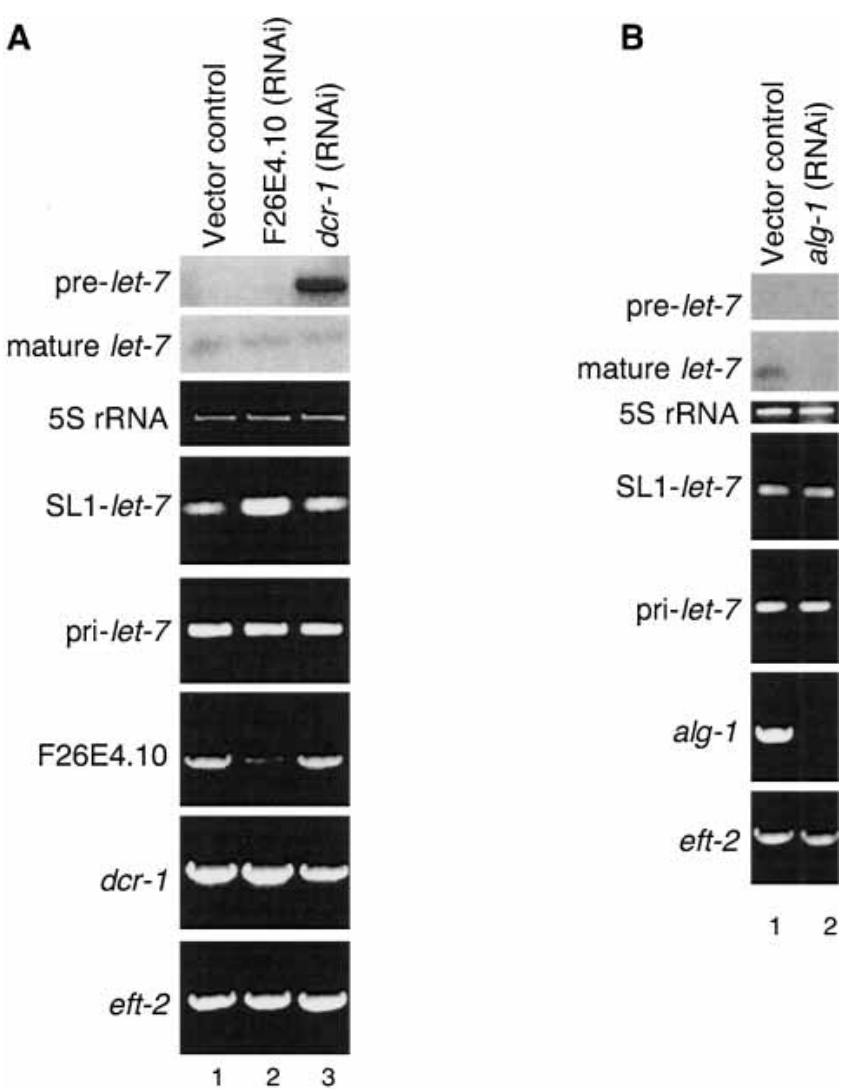

FIGURE 5. Depletion of Drosha, Dicer, or Argonaute homologs inhibits let-7 maturation at independent steps. (A) Total RNA from worms fed vector control (lane 1), F26E4.10 (Drosha) dsRNA (lane 2), or $d c r-1$ (Dicer) dsRNA (lane 3) expressing bacteria was isolated, subjected to Northern analyses to detect precursor and mature let-7 or to oligo dT reverse transcription, followed by PCR amplification of let-7 transcripts, and mRNAs for F26E4.10, dcr-1, and eft-2, as indicated. $5 \mathrm{~S}$ rRNA detected by ethidium bromide staining serves as a control for the Northern analyses. (B) Total RNA from worms fed vector control (lane 1) or alg-1 (argonaute like gene 1) dsRNA (lane 2) expressing bacteria was isolated, subjected to Northern analyses to detect precursor and mature let-7 or to oligo dT reverse transcription, followed by PCR amplification of let-7 transcripts, and mRNAs for alg- 1 and eft-2, as indicated. 5S rRNA detected by ethidium bromide staining serves as a control for the Northern analyses. 
let-7 is a substrate for the Drosha processing pathway in $C$. elegans. Inactivation of F26E4.10 by feeding RNAi produces very mild phenotypes, and we detect little if any decrease in the accumulation of mature let-7 RNA compared to that detected in controls (Fig. 5A, mature let-7 panel). It is possible that there is a limited capacity for the amount of mature let-7 RNA maintained in the cell, and that only a fraction of the primary and precursor substrates generate stable mature RNA. This idea is supported by the observation that relative levels of precursor miRNA in dcr-1-depleted animals can greatly exceed the amount of mature miRNA detected in wild-type animals (Fig. 5A). Additionally, injection of dsRNA targeting F26E4.10 also failed to generate obvious developmental phenotypes that would imply inadequate miRNA processing (data not shown). Although the mRNA levels of F26E4.10 were effectively depleted by RNAi (Fig. 5A), the remaining protein activity is unknown. Thus, it is presently unclear if adequate F26E4.10 activity remains under the RNAi conditions or if other factors compensate in the miRNA biogenesis pathway.

Depletion of $a l g-1$ by RNAi results in a significant reduction in the accumulation of let-7 and lin-4 mature RNAs (Grishok et al. 2001). In the case of lin-4, this defect can be attributed to inefficient processing of pre-lin-4 (Grishok et al. 2001). However, there is no apparent precursor buildup for let-7 (Fig. 5B; Grishok et al. 2001). Because $d c r-1$ (RNAi) causes accumulation of the precursors for both miRNAs (Grishok et al. 2001), there is no evidence that pre-let-7 is particularly unstable in vivo. Our data indicate that transcription and trans-splicing of the initial let-7 RNAs are unaffected in alg-1 (RNAi) (Fig. 5B), ruling out the possibility that reduction of mature let-7 results from significant defects in these processes. Because a variety of mature miRNAs have been found in complexes containing argonaute homologs (Hammond et al. 2001; Hutvagner and Zamore 2002; Mourelatos et al. 2002), these proteins may function primarily to regulate the single-stranded 22-nt forms of miRNAs.

\section{Splicing of let-7 primary transcripts}

Trans-splicing of Spliced Leader (SL) sequences to the $5^{\prime}$ end of mRNAs is a common phenomenon in C. elegans (Blumenthal 1995). Despite the observation that about 70\% of C. elegans pre-mRNAs undergo this process, a definitive function for trans-splicing is yet to be revealed. Nonetheless, deletion of genes encoding the SL1 RNA produces embryonic lethality (Ferguson et al. 1996), and it has been proposed that SL1 trans-splicing may help optimize translation of some mRNAs (Blumenthal 1995). If trans-splicing by SL1 RNA onto some pri-miRNAs influences processing, then miRNA biogenesis could be another important function of trans-splicing in C. elegans.

Conservation of the trans-splice site in C. elegans and C. briggsae and the demonstration that removal of the splice site compromises the rescuing activity of the let-7 gene suggest that trans-splicing may be an important step in the biogenesis of let-7 RNA in C. elegans. What function could trans-splicing serve in the let-7 processing pathway? Sequence or structural alteration of let-7 primary transcripts may be important for subsequent processing steps. The predicted secondary structures produced by folding the entire 890-nt let-7 RNA, or the longer 1731-nt RNA (data not shown), and SL1 spliced version reveal possible structural alterations immediately adjacent to the stem-loop precursor containing the mature RNA (Fig. 3, boxes). Addition of the SL1 22 nt to the $5^{\prime}$ end of the let-7 RNA supports the formation of an extended stem that is initiated by complementary base-pairing between the SL1 sequences and nucleotides encoded by let-7. These predicted structural rearrangements are conserved in the C. briggsae let-7 gene. Additionally, splicing of the let-7 primary transcripts removes sequences that could be inhibitory to the miRNA processing machinery.

Sequences and structures flanking the miRNA hairpin influence maturation to the 22-nt form (Lee et al. 2003; Zeng and Cullen 2003; Chen et al. 2004). Splicing of miRNA primary transcripts could significantly change the nucleotide and structural elements surrounding the hairpin precursor. Although ectopic expression of truncated miRNA transcripts supports production of the mature form of some miRNAs (Zeng and Cullen 2003; Chen et al. 2004), the natural pri-miRNA transcripts may include sequences or structures that regulate expression of specific miRNAs. Thus, identification of endogenous pri-miRNA transcripts is important for understanding how members of this new RNA family are recognized in vivo as substrates for the miRNA processing pathway.

\section{MATERIALS AND METHODS}

\section{RT-PCR analyses}

Total RNA from indicated stages of worm strains was extracted using TRIzol reagent (GIBCO-BRL), including a deoxyribonuclease step (RQ1 DNase, Promega). First-strand cDNA synthesis was performed with $5 \mu \mathrm{g}$ RNA and SUPERSCRIPT II (Invitrogen) reagents, according to the manufacturer's recommendations. RNA ligase-mediated rapid amplification of $5^{\prime}$ and $3^{\prime} \mathrm{cDNA}$ ends (RLM-RACE) was accomplished with the GeneRacer Kit (Invitrogen), using $5 \mu \mathrm{g}$ of total RNA from L3 stage worms. Primer sequences of oligonucleotides used in the RT-PCR reactions are as follows:

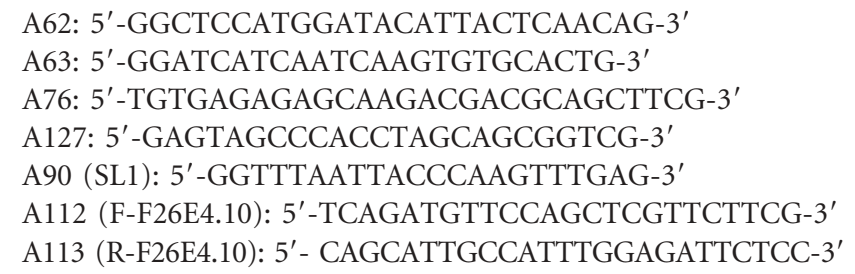


A211 (F- $d c r-1):$ 5'-GCTGCAGATGGAATGTCATTAGAGAGAT TC-3'

A212 (R- $d c r-1): 5^{\prime}$-AGTAGCTTTCGCGATTCGATAGTTCCTT CC-3'

A116 (F-eft-2): 5'-GGTCAACTTCACGGTCGATG-3'

A117 (R-eft-2): 5'-TCCGAGCTTCTCAACAAGAGC-3'

A110 (F-alg-1): 5' -CAAGTGGACCGATTAGTTCGACG-3' A111 (R-alg-1): 5'-TCATTGCTGGGTTGATGGTGATT-3'.

\section{Northern analyses}

Detection of small RNA species ( $<200 \mathrm{nt})$ was carried out by polyacrylamide Northern methods, as previously detailed (Pasquinelli et al. 2003). Analyses of larger RNAs were performed by separating $8 \mu \mathrm{g}$ of total RNA in $1.2 \%$ agarose gels under denaturing conditions. RNA was transferred and UV cross-linked to nylon membranes (Zeta-Probe GT, Biorad), which were then hybridized to PCR-generated DNA probe (A63 + A62) radiolabeled using Prime-It II Random Primer Labeling Kit (Stratagene). Hybridization and wash conditions were performed as previously described (Pasquinelli et al. 2003) except that procedures were carried out at $58^{\circ} \mathrm{C}$.

\section{Transgenics}

For rescue experiments, the wild-type (pAEP19) or 3' splice site deleted (pSEH6) clones were injected at $5 \mathrm{ng} / \mu \mathrm{L}$ with a GFP marker construct (myo-2::yC2.1) into the SP231 (mnDp1(X/V)/+ $\mathrm{V}$; unc-3(e151) let-7(mn112) X) strain (Meneely and Herman 1979). Transgenic, coiling, homozygous let-7(mn112) progeny were scored for rescue: The wild-type construct rescued the lethality in 100/101 of the transgenics; the pSEH6 construct showed no (0/94) rescuing activity. Expression of pri-let-7 from pSEH6 was verified by performing RT-PCR from RNA isolated from transgenic worms; the RNA encoded by the transgene is $187 \mathrm{nt}$ longer than that synthesized by the let-7(mn112) allele and is thus distinguishable from the genomic expression of let-7(mn112).

\section{RNAi}

The rrf-3(pk1426) L4 stage worms were cultured on RNAi plates seeded with bacteria containing vector control plasmid or plasmids expressing double-stranded RNA corresponding to the F26E4.10 or $d c r-1$ or alg-1 genes (Kamath et al. 2003). Progeny from these worms were reared on the same type of RNAi food and collected at the L4 stage of development for RNA isolation and analyses.

\section{ACKNOWLEDGMENTS}

We thank members of our laboratory and J. Posakony, W. McGinnis, J. Dahlberg, and E. Lund for suggestions and critical reading of the manuscript. J.B. and S.H. were supported by funds from the National Institutes of Health (NIH) Cellular and Molecular Biology Graduate Student Training Grant.

The publication costs of this article were defrayed in part by payment of page charges. This article must therefore be hereby marked "advertisement" in accordance with 18 USC section 1734 solely to indicate this fact.

Received June 25, 2004; accepted July 9, 2004.

\section{REFERENCES}

Ambros, V. 2003. MicroRNA pathways in flies and worms: Growth, death, fat, stress, and timing. Cell 113: 673-676.

Ambros, V., Lee, R.C., Lavanway, A., Williams, P.T., and Jewell, D. 2003. MicroRNAs and other tiny endogenous RNAs in C. elegans. Curr. Biol. 13: 807-818.

Aravin, A.A., Lagos-Quintana, M., Yalcin, A., Zavolan, M., Marks, D., Snyder, B., Gaasterland, T., Meyer, J., and Tuschl, T. 2003. The small RNA profile during Drosophila melanogaster development. Dev. Cell 5: 337-350.

Aukerman, M.J. and Sakai, H. 2003. Regulation of flowering time and floral organ identity by a MicroRNA and its APETALA2-like target genes. Plant Cell 15: 2730-2741.

Bartel, D.P. 2004. MicroRNAs: Genomics, biogenesis, mechanism, and function. Cell 116: 281-297.

Basyuk, E., Suavet, F., Doglio, A., Bordonne, R., and Bertrand, E. 2003. Human let-7 stem-loop precursors harbor features of RNase III cleavage products. Nucleic Acids Res. 31: 6593-6597.

Blumenthal, T. 1995. Trans-splicing and polycistronic transcription in Caenorhabditis elegans. Trends Genet. 11: 132-136.

Bohnsack, M.T., Czaplinski, K., and Gorlich, D. 2004. Exportin 5 is a RanGTP-dependent dsRNA-binding protein that mediates nuclear export of pre-miRNAs. RNA 10: 185-191.

Cerutti, L., Mian, N., and Bateman, A. 2000. Domains in gene silencing and cell differentiation proteins: The novel PAZ domain and redefinition of the Piwi domain. Trends Biochem. Sci. 25: 481-482.

Chen, C.Z., Li, L., Lodish, H.F., and Bartel, D.P. 2004. MicroRNAs modulate hematopoietic lineage differentiation. Science 303: 8386.

Feinbaum, R. and Ambros, V. 1999. The timing of lin-4 RNA accumulation controls the timing of postembryonic developmental events in Caenorhabditis elegans. Dev. Biol. 210: 87-95.

Ferguson, K.C., Heid, P.J., and Rothman, J.H. 1996. The SL1 transspliced leader RNA performs an essential embryonic function in Caenorhabditis elegans that can also be supplied by SL2 RNA. Genes \& Dev. 10: 1543-1556.

Grad, Y., Aach, J., Hayes, G.D., Reinhart, B.J., Church, G.M., Ruvkun, G., and Kim, J. 2003. Computational and experimental identification of C. elegans microRNAs. Mol. Cell 11: 1253-1263.

Grishok, A., Pasquinelli, A.E., Conte, D., Li, N., Parrish, S., Ha, I., Baillie, D.L., Fire, A., Ruvkun, G., and Mello, C.C. 2001. Genes and mechanisms related to RNA interference regulate expression of the small temporal RNAs that control C. elegans developmental timing. Cell 106: 23-34.

Hammond, S.M., Boettcher, S., Caudy, A.A., Kobayashi, R., and Hannon, G.J. 2001. Argonaute2, a link between genetic and biochemical analyses of RNAi. Science 293: 1146-1150.

Hutvagner, G. and Zamore, P.D. 2002. A microRNA in a multipleturnover RNAi enzyme complex. Science 297: 2056-2060.

Hutvagner, G., McLachlan, J., Pasquinelli, A.E., Balint, E., Tuschl, T., and Zamore, P.D. 2001. A cellular function for the RNA-interference enzyme Dicer in the maturation of the let-7 small temporal RNA. Science 293: 834-838.

Johnson, S.M., Lin, S.Y., and Slack, F.J. 2003. The time of appearance of the C. elegans let-7 microRNA is transcriptionally controlled utilizing a temporal regulatory element in its promoter. Dev. Biol. 259: $364-379$.

Johnston, R.J. and Hobert, O. 2003. A microRNA controlling left/right neuronal asymmetry in Caenorhabditis elegans. Nature 426: 845849.

Kamath, R.S., Fraser, A.G., Dong, Y., Poulin, G., Durbin, R., Gotta, M., Kanapin, A., Le Bot, N., Moreno, S., Sohrmann, M., et al. 2003. 
Systematic functional analysis of the Caenorhabditis elegans genome using RNAi. Nature 421: 231-237.

Ketting, R.F., Fischer, S.E., Bernstein, E., Sijen, T., Hannon, G.J., and Plasterk, R.H. 2001. Dicer functions in RNA interference and in synthesis of small RNA involved in developmental timing in $C$. elegans. Genes \& Dev. 15: 2654-2659.

Lagos-Quintana, M., Rauhut, R., Yalcin, A., Meyer, J., Lendeckel, W., and Tuschl, T. 2002. Identification of tissue-specific microRNAs from mouse. Curr. Biol. 12: 735-739.

Lagos-Quintana, M., Rauhut, R., Meyer, J., Borkhardt, A., and Tuschl, T. 2003. New microRNAs from mouse and human. RNA 9: 175179.

Lai, E.C. 2003. MicroRNAs: Runts of the genome assert themselves. Curr. Biol. 13: R925-936.

Lai, E.C., Tomancak, P., Williams, R.W., and Rubin, G.M. 2003. Computational identification of Drosophila microRNA genes. Genome Biol. 4: R42.

Lee, R.C., Feinbaum, R.L., and Ambros, V. 1993. The C. elegans heterochronic gene lin-4 encodes small RNAs with antisense complementarity to lin-14. Cell 75: 843-854.

Lee, Y., Jeon, K., Lee, J.T., Kim, S., and Kim, V.N. 2002. MicroRNA maturation: Stepwise processing and subcellular localization. EMBO J. 21: 4663-4670.

Lee, Y., Ahn, C., Han, J., Choi, H., Kim, J., Yim, J., Lee, J., Provost, P., Radmark, O., Kim, S., et al. 2003. The nuclear RNase III Drosha initiates microRNA processing. Nature 425: 415-419.

Lim, L.P., Glasner, M.E., Yekta, S., Burge, C.B., and Bartel, D.P. 2003 a. Vertebrate microRNA genes. Science 299: 1540.

Lim, L.P., Lau, N.C., Weinstein, E.G., Abdelhakim, A., Yekta, S., Rhoades, M.W., Burge, C.B., and Bartel, D.P. 2003b. The microRNAs of Caenorhabditis elegans. Genes \& Dev. 17: 991-1008.

Lund, E., Guttinger, S., Calado, A., Dahlberg, J.E., and Kutay, U. 2004. Nuclear export of microRNA precursors. Science 303: 95-98.

Meneely, P.M. and Herman, R.K. 1979. Lethals, steriles and deficiencies in a region of the $\mathrm{X}$ chromosome of Caenorhabditis elegans. Genetics 92: 99-115.

Mourelatos, Z., Dostie, J., Paushkin, S., Sharma, A., Charroux, B., Abel, L., Rappsilber, J., Mann, M., and Dreyfuss, G. 2002. miRNPs:
A novel class of ribonucleoproteins containing numerous microRNAs. Genes \& Dev. 16: 720-728.

Pasquinelli, A.E. and Ruvkun, G. 2002. Control of developmental timing by microRNAs and their targets. Annu. Rev. Cell Dev. Biol. 18: 495-513.

Pasquinelli, A.E., Reinhart, B.J., Slack, F., Martindale, M.Q., Kuroda, M.I., Maller, B., Hayward, D.C., Ball, E.E., Degnan, B., Muller, P., et al. 2000. Conservation of the sequence and temporal expression of let-7 heterochronic regulatory RNA. Nature 408: 86-89.

Pasquinelli, A.E., McCoy, A., Jimenez, E., Salo, E., Ruvkun, G., Martindale, M.Q., and Baguna, J. 2003. Expression of the 22 nucleotide let-7 heterochronic RNA throughout the Metazoa: A role in life history evolution? Evol. Dev. 5: 372-378.

Reed, R. 2003. Coupling transcription, splicing and mRNA export. Curr. Opin. Cell Biol. 15: 326-331.

Reinhart, B.J., Slack, F.J., Basson, M., Pasquinelli, A.E., Bettinger, J.C., Rougvie, A.E., Horvitz, H.R., and Ruvkun, G. 2000. The 21nucleotide let-7 RNA regulates developmental timing in Caenorhabditis elegans. Nature 403: 901-906.

Schwarz, D.S. and Zamore, P.D. 2002. Why do miRNAs live in the miRNP? Genes \& Dev. 16: 1025-1031.

Simmer, F., Tijsterman, M., Parrish, S., Koushika, S.P., Nonet, M.L., Fire, A., Ahringer, J., and Plasterk, R.H. 2002. Loss of the putative RNA-directed RNA polymerase RRF-3 makes $C$. elegans hypersensitive to RNAi. Curr. Biol. 12: 1317-1319.

Smalheiser, N.R. 2003. EST analyses predict the existence of a population of chimeric microRNA precursor-mRNA transcripts expressed in normal human and mouse tissues. Genome Biol. 4: 403.

Yi, R., Qin, Y., Macara, I.G., and Cullen, B.R. 2003. Exportin-5 mediates the nuclear export of pre-microRNAs and short hairpin RNAs. Genes \& Dev. 17: 3011-3016.

Zeng, Y. and Cullen, B.R. 2003. Sequence requirements for micro RNA processing and function in human cells. RNA 9: 112-123.

Zeng, Y., Wagner, E.J., and Cullen, B.R. 2002. Both natural and designed micro RNAs can inhibit the expression of cognate mRNAs when expressed in human cells. Mol. Cell 9: 1327-1333.

Zuker, M. 2003. Mfold web server for nucleic acid folding and hybridization prediction. Nucleic Acids Res. 31: 3406-3415. 

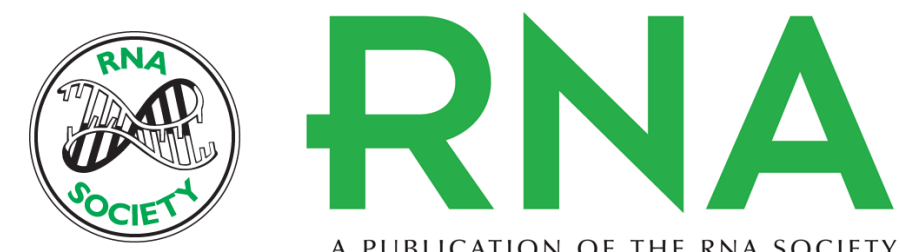

A PUBLICATION OF THE RNA SOCIETY

\section{Trans-splicing and polyadenylation of let-7 microRNA primary transcripts}

JOHN BRACHT, SHAUN HUNTER, RACHEL EACHUS, et al.

RNA 2004 10: 1586-1594

References This article cites 45 articles, 18 of which can be accessed free at: http://rnajournal.cshlp.org/content/10/10/1586.full.html\#ref-list-1

License

Email Alerting Receive free email alerts when new articles cite this article - sign up in the box at the top Service right corner of the article or click here.

To subscribe to RNA go to:

http://rnajournal.cshlp.org/subscriptions 